\title{
Análisis comparativo de la red de flujos de conocimiento e información tecnológica en dos regiones líderes en el cultivo de camarón en México*
}

\author{
Jorge I. León Balderramaa - Lydia V. Gutiérrez López \\ - J. Crisóforo Carrazco Escalantec
}

\begin{abstract}
Resumen: El presente estudio tiene por objetivo "reconstruir" y comparar las redes de flujo de conocimiento e información tecnológica (RFCIT) en la industria acuícola, específicamente en el cultivo de camarón, de dos de las principales regiones productoras de camarón de cultivo en México: el municipio de Ahome, ubicado al norte del Estado de Sinaloa, y la región costera centro-sur del estado de Sonora. La investigación se basa en el enfoque del análisis de redes sociales y la información fue obtenida mediante la aplicación de una encuesta a una muestra de 91 empresas dedicadas al cultivo de camarón en las regiones mencionadas. Las conclusiones del estudio señalan que las características y la configuración de las RFCIT de la acuicultura de Sinaloa le han permitido a esta entidad tener una ventaja de competitividad frente a Sonora, para paliar los efectos de la crisis de los últimos años.
\end{abstract}

Palabras clave: Acuacultura; análisis de redes sociales; redes empresas-proveedores de conocimiento.

Fecha de recibido: 25 de mayo de 2018 Fecha de aprobado: 15 de febrero de 2019

Cómo citar: León Balderrama, J. I., Gutiérrez López, L. V. y Carrazco Escalante, J. C. (2019). Análisis comparativo de la red de flujos de conocimiento e información tecnológica en dos regiones líderes en el cultivo de camarón en México. Revista Facultad de Ciencias Económicas, 27(2), 9-32. rev.fac.cienc. econ. doi: https://doi.org/10.18359/rfce.3953

JEL: L14, M11, Q22

* Artículo de investigación derivado del proyecto Redes de transferencia de conocimiento y su impacto en la innovación en las plantas acuícolas de Sonora y Sinaloa, 2015-2016, financiado por la Coordinación de Desarrollo Regional del Centro de Investigación en Alimentación y Desarrollo, A.C. México (Conacyt).

a Doctor en Ciencias Sociales de la Universidad Autónoma de Sinaloa, México. Investigador titular en el Centro de Investigación en Alimentación y Desarrollo A.C., coordinador del Área de Desarrollo Regional. Dirección postal: carretera a La Victoria km 0.6 S/N, Col. Ejido La Victoria, CP. 83304, Hermosillo, México. Tel. (52) 6622892400 ext. 370. Correo electrónico: jleon@ciad.mx

b Doctora en Ciencias y especialista en Desarrollo Regional. Centro de Investigación en Alimentación y Desarrollo A.C., coordinadora del Área de Desarrollo Regional. Correo electrónico: venecy14@gmail.com

c Doctor en Ciencias y especialista en Desarrollo Regional. Centro de Investigación en Alimentación y Desarrollo A.C., Coordinador del Área de Desarrollo Regional. Correo electrónico:

hechoenmexico86@hotmail.com 


\title{
Comparative Analysis of Networks of Knowledge Flow and Technological Information in Two Leading Shrimp-Farming Regions in Mexico
}

\begin{abstract}
The purpose of this study is to "reconstruct" and compare the networks of knowledge flow and technological information (NKFTI) in the aquaculture industry, specifically in shrimp farming in two of the main regions producing farmed-shrimp in Mexico: The municipality of Ahome, located to the north of Sinaloa, and the coastal south-center region of Sonora. Research is based on the approach to the analysis of social networks and information gathered by conducting a survey with a sample of 91 companies dedicated to shrimp farming in the regions mentioned above. The study findings show that the features and settings of the NKFTI of Sinaloa aquaculture have allowed this region to gain a competitive advantage over Sonora, to mitigate the effects of the crisis in recent years.
\end{abstract}

Keywords: Aquaculture; social network analysis, company-supplier networks of knowledge.

\section{Análise comparativa da rede de fluxos de conhecimento e informações tecnológicas em duas regiões líderes no cultivo de camarão no México}

Resumo: O objetivo deste estudo é "reconstruir" e comparar as redes de fluxo de conhecimento e informação tecnológica (RFCIT) no setor aquícola, especificamente na criação de camarões, de duas das principais regiões produtoras de criação de camarões no México: o município de Ahome, localizado ao norte do estado de Sinaloa, e o litoral centro-sul do estado de Sonora. A pesquisa tem como base a análise de redes sociais, as informações foram obtidas a partir da aplicação de uma enquete a uma amostra de 91 empresas dedicadas à criação de camarão nas regiões mencionadas. As conclusões do estudo indicam que as características e a configuração das RFCIT da aquicultura em Sinaloa permitiram que ela tivesse uma vantagem competitiva sobre Sonora, mitigando os efeitos da crise dos últimos anos.

Palavras-chave: Aquicultura; análise de redes sociais; fornecedores de conhecimento de redes de negócios. 


\section{Introducción}

La acuacultura es considerada una de las actividades con mayor potencial de desarrollo en México. En los últimos años, se ha convertido en una de las alternativas con mayor viabilidad económica para la producción de alimentos en algunas regiones del país. La relevancia de esta actividad está relacionada con sus impactos sociales generales, entre los que destaca que la acuacultura: a) es una fuente importante de empleos en las comunidades costeras, ya que ofrece trabajos en regiones con pocas oportunidades de obtenerlos (se estima que actualmente genera 15.000 empleos directos en Sinaloa y Sonora); b) reduce la migración a las zonas urbanas; c) disminuye el esfuerzo pesquero, y d) es una importante generadora de divisas. Sin embargo, al igual que en otros países, esta actividad ha enfrentado fuertes crisis en los últimos años debido a los impactos de enfermedades como la mancha blanca y la muerte temprana, que han afectado de manera drástica la producción.

Sonora y Sinaloa sobresalen por ser las entidades federativas mexicanas con el mayor aporte en la producción acuícola a nivel nacional. La aportación de estas dos entidades federativas a la producción acuícola es muy destacada, especialmente en lo que respecta a la producción de camarón de cultivo. Hacia 2010, las dos entidades juntas contribuían con el $90 \%$ de la producción en volumen, produciendo 118.000 toneladas de un total nacional de 132.000. Sin embargo, en el periodo 2010-2013 la producción nacional de camarón de cultivo sufrió un decremento de más del $50 \%$, al pasar de más de 132.000 toneladas a 60.000, aproximadamente, en el periodo señalado. Estas entidades también han sentido los impactos negativos de esta crisis sectorial, en especial Sonora, que pasó de una producción de 84.000,8 toneladas en 2009 a 14.000,6 en 2013. Aunado a estas dificultades que ha presentado el crecimiento de la actividad en la región noroeste de México en los años recientes, también ha estado en entredicho la sustentabilidad de los sistemas acuícolas que son empleados actualmente en la región. Estudios sobre el tema han documentado diferentes efectos adversos que comprometen la sustentabilidad de la acuacultura en la zona costera del golfo de California (Del Río Salas, Martínez Durazo y Jara Marini, 2016; BerlangaRobles, Ruiz-Luna y Hernández-Guzmán, 2011); por ejemplo, conflicto con otras actividades económicas (como agricultura, pesquerías y turismo), el cambio de uso de suelo, la deforestación de manglares y diferentes tipos de contaminación (principalmente asociada a la descarga y filtración de materia orgánica).

Con las condiciones adversas presentadas en los últimos años, en especial la crisis y el declive productivo, los productores han tenido que tomar decisiones difíciles, como postergar inversiones y adaptarse muy rápido al manejo, la tecnología y la innovación. El cultivo de camarón es una actividad que requiere constante actualización tecnológica, los productores utilizan la tecnología para mejorar los rendimientos, combatir las enfermedades y reducir los costos, básicamente. Entre las principales nuevas tecnologías que desde la última década se están tratando de introducir, con diferentes niveles de éxito, destacan las siguientes: vacunas y antibióticos, alimentos mejorados, uso de probióticos en el agua, desinfección de aguas en estanque, probióticos e inmuno-estimulantes en alimentos, manipulación de dinámicas de estanques, reducción de intercambios de aguas, filtración mejorada, reducción de proteínas en alimentos, empleo de sustratos artificiales, revestimiento de estanques, uso de policultivos con otras especies y mitigación medioambiental.

La información, las ideas y el conocimiento acerca de estas y otras tecnologías las pueden obtener los productores de múltiples fuentes externas. Diversos agentes e instituciones logran jugar un papel clave en el desarrollo científico y tecnológico de este sector, como son las universidades y los tecnológicos, los centros de investigación, los comités de sanidad, proveedores, competidores, clientes, consultores privados, etc.

El objetivo de esta investigación es contribuir al estudio empírico del papel clave que juegan las interacciones sociales, las cuales implican 
intercambios de conocimiento entre las empresas y los diversos agentes, máxime cuando se trata de conocimiento de corte tecnológico que tiene implicaciones sobre procesos de innovación y de actualización tecnológica en las empresas acuícolas; esto, partiendo del estudio en particular de la acuicultura de camarón desarrollada en las principales regiones productoras de Sonora y Sinaloa. El estudio tiene los siguientes objetivos específicos: a) identificar la conformación de la red de transferencia de conocimientos que se establece entre las empresas acuícolas y sus fuentes de conocimiento científico y tecnológico (otras empresas, consultores, organismos gubernamentales, universidades, centros de investigación, proveedores, competidores, organizaciones de la sociedad civil, etc.) para el caso de la región centro-sur de Sonora y la región acuícola de Ahome en Sinaloa; b) caracterizar la red de transferencia de conocimiento en la industria acuícola en términos de tamaño, estructura y densidad, y hacer una comparación entre las dos entidades estudiadas, y c) estimar variables relacionales de las empresas acuícolas, como aquellas asociadas a la posición que ocupan dentro de la red: nivel de centralidad, nivel de intermediación y nivel de cercanía, etc., y otras que tienen que ver con las características de las relaciones que establecen con otros agentes de la red, como la fuerza y la diversidad de las vinculaciones.

El resto del artículo se estructura de la siguiente manera. En el primer apartado se revisa la literatura sobre la importancia que tienen las redes de conocimiento en los procesos de innovación tecnológica; en el segundo, se destaca la utilidad y las ventajas que posee el análisis de redes sociales (ARs) en el estudio de la innovación tecnológica; en el tercero se describe la metodología de la investigación y el marco analítico que vamos a utilizar en nuestro análisis. En el quinto apartado se presentan los resultados del análisis y la discusión sobre estos. El último apartado contiene las conclusiones del estudio y, al final, se enlistan las referencias utilizadas para apoyar este trabajo de investigación.

\section{La importancia de las redes de flujo de conocimiento e información tecnológica en los procesos de innovación y mejora tecnológica}

El conocimiento y la innovación se han convertido en recursos fundamentales en la era actual de la economía del conocimiento. La innovación constituye un factor clave en la competitividad de las empresas; sin embargo, la capacidad empresarial de innovar se ve condicionada por factores internos y externos, en un contexto de competencia cada vez más exigente. En particular, las empresas de sectores primarios enfrentan grandes limitaciones para consolidar sus propios departamentos de investigación y desarrollo ( $\mathrm{I}+\mathrm{D})$, por lo que deben recurrir a fuentes externas de conocimiento para complementar sus recursos internos. Del mismo modo, la complejidad y la variabilidad de la tecnología y de los mercados incrementan la necesidad de fuentes externas que complementan el conocimiento (Caloghirou, Kastelli y Tsakanikas, 2004; Enkel, Gasmann y Chesbrough, 2009). Las empresas de sectores alimentarios también se están adaptando a la nueva realidad con sus estrategias competitivas, basándose en la innovación y la adopción de nuevas tecnologías. Específicamente, se impone cada vez más una estrategia dual, al hacer uso de diversas fuentes de conocimiento y tecnología internas y externas para incrementar la velocidad de los desarrollos. De esta manera, las relaciones y la cooperación con actores externos como fuentes de conocimiento, ideas y tecnologías se vuelven una opción de interés a causa de los riesgos en la inversión en $\mathrm{I}+\mathrm{D}$, los cuales pueden ser compartidos mediante la asociación con otros organismos.

La innovación basada en procesos de colaboración e intercambio tiene su base en los modelos interactivos de innovación (Baldwin y Von Hippel, 2011). Estos modelos destacan el carácter interactivo-colectivo del proceso de innovación, lo que sugiere que los innovadores dependen en 
gran medida de su interacción con los usuarios y proveedores, y con una serie de instituciones dentro del sistema de innovación (Lundvall, 2010). El enfoque de los sistemas de innovación destaca el papel de las fuentes externas de conocimiento como un factor clave del aprendizaje interactivo.

El impacto que tienen la colaboración y las relaciones que implican flujos e intercambio de conocimiento con agentes externos en la innovación poco se ha analizado en México; no obstante, en otros países, por ejemplo, Estados Unidos, Canadá y algunos de Europa, varios autores han analizado las fuentes de conocimiento utilizadas por las empresas y el impacto que tienen en el desempeño competitivo-innovador de estas (Maffini Gomes y Kruglianskas, 2009; Cricelli y Grimaldi, 2010). Otros se han enfocado en el impacto de la colaboración y han detectado que la innovación y las nuevas ideas son enriquecidas a través de la diversa búsqueda de información (Powell, Koput y Smith-Doerr, 1996; Caloghirou et al., 2004; Laursen y Salter, 2006).

\section{El enfoque de análisis de redes sociales en el estudio de la innovación tecnológica en las empresas}

Las redes de innovación y de transferencia de tecnología han sido estudiadas con base en la perspectiva del ARs, enfoque que permite analizar las interacciones entre individuos mediante la configuración de sus estructuras. Borgatti, Mehra, Brass y Labianca (2009) consideran el ARs un cuerpo de aspectos teóricos y no solo un conjunto de métodos. El ARs difiere de enfoques tradicionales de ciencias sociales en varios aspectos, y uno de ellos radica en los datos de análisis que se emplean. La mayoría de los enfoques tradicionales utilizan como datos las variables o atributos de sus unidades de análisis, considerando que se parte de “¿qué se quiere conocer?"; en cambio, el ARs parte de " $¿ a$ quién conoces?", y esto conlleva el uso de datos relacionales y no solo atributivos de los actores.

En estudios sobre innovación, una ventaja que ofrece el ARs respecto a otros enfoques es que permite utilizar los datos relacionales de los actores y las características estructurales de la red como elementos de análisis de las relaciones entre actores, que a su vez son dependientes de otras interacciones. En el ARs esta dependencia está correlacionada con los atributos de cada actor y de su comportamiento. El A Rs ayuda a sobrellevar problemas relacionados con la cooperación y la confianza dentro de un grupo de actores mediante el establecimiento de niveles de fortaleza de las relaciones entre los actores. Por otra parte, la influencia y el poder de ciertos actores o individuos en la red pueden ser analizados por medio de la diversidad e interdependencia de las relaciones. Lo anterior lleva a un análisis más profundo en cuanto a cómo y con quiénes se relacionan las empresas con mayores resultados innovadores. Así, el ARs juega un importante rol en el estudio de la evaluación de impactos, ya que genera indicadores reticulares a nivel de las empresas y de la red de innovación, los cuales pueden ser utilizados en análisis que miden el impacto en innovación (Van der Valk y Gijsbers, 2010).

El tamaño y la composición de una red, la densidad y su centralidad son aspectos muy importantes cuando se discute sobre el impacto en la innovación (Liu y Chaminade, 2010). El tamaño y la forma de una red son características muy importantes, ya que determinan la estructura de las relaciones porque cada empresa dispone de recursos limitados para crear y mantener relaciones. Asimismo, la densidad de la red es comúnmente medida en términos del número de relaciones directas entre los actores de la red y tiene implicaciones importantes en el acceso a competencias, en la gestión de las redes y, por lo tanto, en la innovación (Cruz Gomes, Ribeiro Galina, Oliveira do Prado y Silveira Porto, 2017; Choi, Sang-Hyun y Cha, 2013).

Varios autores han analizado la estructura de las redes de innovación con base en la densidad de sus relaciones. Por ejemplo, Hansen, Mors y Lovas (2005) y Oh, Labianca y Chung (2006) demuestran que las redes de alta densidad tienden a reforzar la redundancia del conocimiento y pueden ser opuestas a la innovación; mientras que McCarthy (2008) llegó a una conclusión opuesta al 
demostrar que una red densa puede ayudar a difundir normas institucionales de comportamiento y también facilita el traslado de la información, cuestiones sumamente importantes para la innovación. De igual forma, Reagans y McEvil (2003) señalan cómo la cohesión, en términos de mayor cantidad de lazos directos y el tamaño de la red, tiene un fuerte impacto en la transferencia de conocimientos.

La posición de los actores en la red puede ser analizada mediante medidas de centralidad; además, tiene implicaciones importantes para el aprendizaje y la innovación, ya que influye, por ejemplo, en la participación de otros actores en la red, en el poder de negociación de los diferentes actores o en el control de la información (Nooteboom, 2004; Choi et al., 2013). La centralidad en la red es una de las características más analizadas desde la perspectiva del ARs, es el reflejo de la posición de un actor y es medida en función del número de vínculos o por el poder de influencia en la red. Mayor centralidad en la red significa un mayor número de vínculos frecuentes (Borgatti, Everett y Freeman, 2002). La centralidad puede ser medida en función de diversos indicadores, como el número de conexiones directas (grado), la frecuencia con que un actor conecta a otros actores con base en distintas vías (intermediación) y la centralidad de cercanía mediante su grado de proximidad con respecto a todos los otros individuos. El número de relaciones directas (grado) que mantiene un actor es una de las medidas de centralidad más básicas e intuitivas del ARs.

Teóricamente, podemos esperar que el posicionamiento de los actores individuales y la forma como están conectados a otros actores de la red tengan también implicaciones importantes para la innovación tecnológica. Contar con un posicionamiento central, hacer la función de puente en la red, tener una estrecha relación con un actor que posee varias conexiones son condiciones que les sirven a las empresas para asegurar el acceso a recursos críticos (Wang, Zhao, Li y Li, 2015; Scott, 2000; Nooteboom, 2004). Por el contrario, una posición periférica en la red, que implica la necesidad de ir a través de muchos nodos para acceder a la información relevante, es una condición particular que podría obstaculizar gravemente el flujo de conocimiento y, por lo tanto, la innovación dentro de la red.

La fuerza de los vínculos es otra de las características principales de las relaciones que se dan en una red, que además ha sido medida en términos de frecuencia o de confianza. Hay una gran cantidad de literatura sobre las ventajas de los vínculos débiles (no intensos) contra los vínculos fuertes (intensos) en redes, pero los hallazgos de los estudios no son concluyentes respecto a los efectos netos de la intensidad de los vínculos sobre los procesos de innovación (Rost, 2011). En el lado positivo de todo esto, las redes de vínculos débiles pueden dar acceso a información nueva y no redundante e introducir flexibilidad en la misma red, mientras que los vínculos fuertes pueden facilitar el intercambio de información compleja; pero estos últimos también pueden tener implicaciones negativas, como las situaciones de falta de renovación de la ideas y de conocimientos (D'Costa, 2002; Narula, 2002). Evidentemente, si los vínculos fuertes o débiles resultan ser más favorable es una cuestión que depende de las características del aspecto (la "materia") que tiene que ser transferido; mientras que los lazos fuertes pueden ser más adecuados para el intercambio de conocimientos complejos, los lazos débiles podrían ser más beneficiosos para la búsqueda de información (Hansen, 1999). La fuerza de un vínculo es una combinación de la cantidad de tiempo, la intensidad emocional, la intimidad y los servicios recíprocos que caracterizan a ese vínculo.

En lo que respecta a la diversidad de las relaciones, Booher e Innes (2002) analizan el efecto en resultados de innovación y detectan que tiene una enorme influencia. De igual forma, otros autores han argumentado que la innovación de tipo radical y las nuevas ideas son enriquecidas a través de la búsqueda de información en diversas fuentes (Laursen y Salter, 2006). Por su parte, Fritsch y Kauffeld (2010) sostienen que la diversidad es un buen indicador de la "no redundancia" de la información. 


\section{El método. Información y procedimientos para el análisis empírico de las redes de flujo de conocimiento e información tecnológica en el cultivo de camarón en las regiones de Ahome, Sinaloa y el centro-sur de Sonora}

En este apartado se describen las características principales de la encuesta que se llevó a cabo para obtener información necesaria para el análisis; además, se explican los procedimientos específicos del ARs que fueron aplicados para el procesamiento de la información conseguida, como la elaboración de sociogramas, la estimación de medidas globales de las redes y la estimación de medidas locales de posición y naturaleza de las relaciones.

\section{Encuesta sobre redes de provisión de conocimiento e información tecnológica de empresas dedicadas al cultivo de camarón en el noroeste de México}

La información necesaria para el análisis se consiguió a través de una encuesta hecha a 91 plantas dedicadas al cultivo de camarón, 58 pertenecientes a la región de Ahome Sinaloa y 33 a la región centro sur del Estado de Sonora, distribuidas estas últimas de la siguiente manera: 12 de Bahía de Kino, 2 de Tastiota, 7 de El Cardonal, 2 de Cruz de Piedra, 2 de Bahía de Lobos, 2 de Los Mélagos, 1 de El Siari, 1 de Santa Bárbara, 2 de La Atanasia, 1 de El Tobari y 1 de El Riíto (figura 1). La aplicación de la encuesta se llevó a cabo en el periodo agosto-noviembre de 2015 en Sonora y enero-mayo de 2016 en Sinaloa.

En el ARs se emplean técnicas de muestreo distintas a los análisis empíricos convencionales. La muestra debe ser representativa, pero no en el sentido estadístico. En el ARs no se conoce a priori el tamaño de la población (el tamaño de las redes de flujo de conocimiento e información tecnológica [RFCIT]), por lo que resulta imposible conocer de manera anticipada la integración global de las dos RFCIT que se pretenden comparar en este estudio. El objetivo del muestreo consiste en contar con una subred que represente a la red total, es decir, que preserve ciertas propiedades de la red original. Como lo han señalado Leskovec y Faloutsos (2006), los métodos de muestreo en ARs se pueden dividir en tres grupos: métodos basados en la selección aleatoria de nodos, métodos basados en la selección aleatoria de vínculos y técnicas de exploración que simulan caminatas aleatorias o la propagación de virus para encontrar nodos simples representativos. En este estudio se emplea la técnica de muestreo más básica, el muestro aleatorio de nodos.
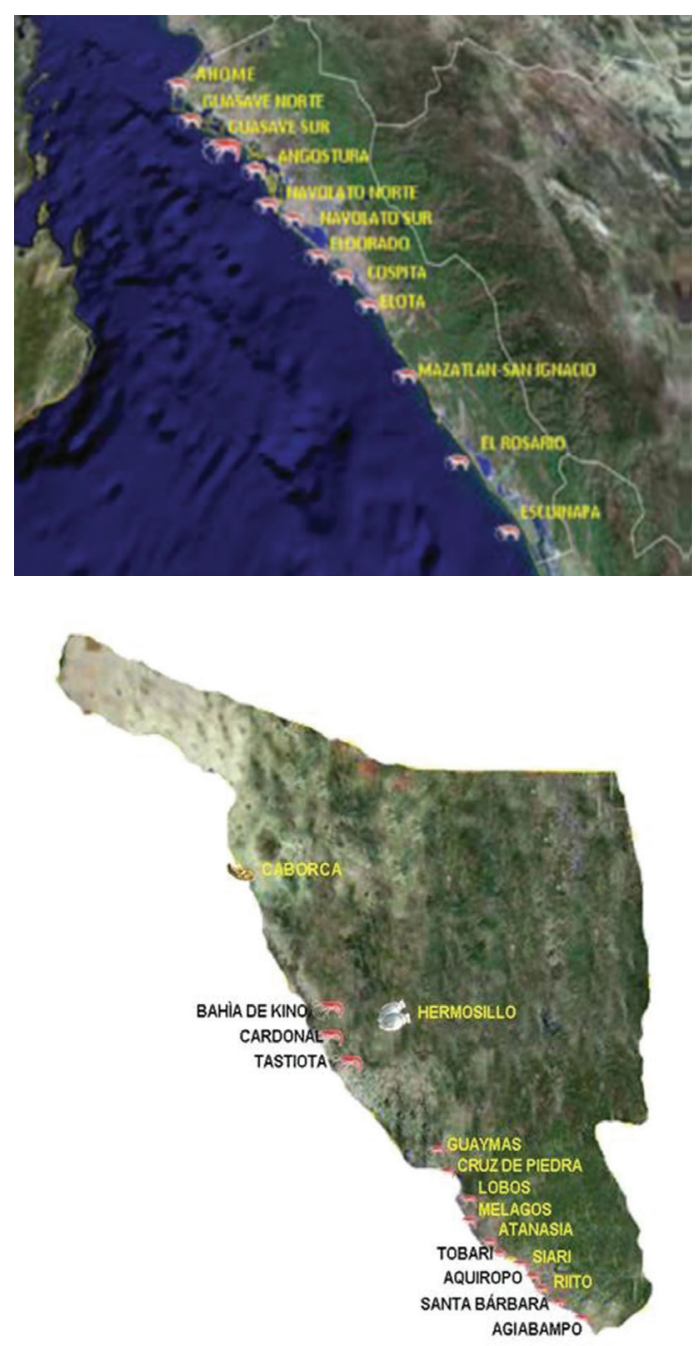

Figura 1. Ubicación de las regiones productoras de camarón de cultivo estudiadas

Fuente: elaboración propia. 
La red muestreada contiene las 91 plantas acuícolas seleccionadas en las dos regiones y todos los enlaces entre ellas y sus proveedores de información técnica. El muestreo aleatorio de nodos se utiliza, entre otras, cuando se selecciona por primera vez una muestra de individuos y luego se observa su comportamiento de contacto. Numerosas encuestas y recopilaciones de datos utilizan este método, por ejemplo, Mastrandrea, Fournet, Barrat (2015), que miden el patrón de contacto entre estudiantes de secundaria que usan sensores portátiles.

El cuestionario utilizado se diseñó para conseguir información reticular; es decir, para identificar las relaciones que implican la transferencia y el intercambio de información y conocimiento relacionado con las nuevas tecnologías del ramo

Recuadro 1. Cuestionario aplicado en la Encuesta sobre redes de provisión de conocimiento e información tecnológica de empresas dedicadas al cultivo de camarón en el noroeste de México

1. Por favor, enliste cada una de las fuentes externas de conocimiento e información con las que se ha relacionado para realizar mejoras o desarrollos tecnológicos en productos, procesos o servicios en su empresa. Use las líneas que considere necesarias de acuerdo con la experiencia de su empresa.

2. Una vez que haya escrito el nombre o razón social de cada una de sus fuentes de conocimiento e información tecnológica, por favor de marcar en la primera columna el tipo de organización, de acuerdo con la siguiente clasificación: 1 = proveedor, $2=$ competidor, 3 = universidad $/ \mathrm{CI}, 4=$ cliente, 5 = consultor, $6=$ organismo de gobierno, $7=$ laboratorio privado, $8=$ organismo 0 asociación civil y $9=$ otros.

3. Enseguida evalúe la importancia de cada fuente teniendo en cuenta los criterios de las columnas $A, B$ y $C$, y la siguiente escala de importancia: $1=$ muy poca, 2 = poca, $3=$ media, $4=$ alta y $5=$ muy alta.

\begin{tabular}{|c|c|c|c|c|c|c|c|c|c|c|}
\hline \multicolumn{2}{|c|}{ Nombre y giro } & \multicolumn{3}{|l|}{ A } & \multicolumn{2}{|l|}{ B } & \multicolumn{4}{|l|}{ C } \\
\hline & & $\begin{array}{l}\text { Importancia se } \\
\text { cantidad de inf }\end{array}$ & & $\begin{array}{l}\text { la } \\
\text { ación }\end{array}$ & $\begin{array}{l}\text { Importancia seg } \\
\text { a las mejoras int }\end{array}$ & $\begin{array}{l}\text { su aporte } \\
\text { ducidas }\end{array}$ & $\begin{array}{l}\text { Importancia } \\
\text { frecuencia d }\end{array}$ & & ún la & cación \\
\hline 1 & $\begin{array}{lll}1 \square & 2 \square & 3 \square \\
4 \square & 5 \square & 6 \square \\
7 \square & 8 \square & 9 \square\end{array}$ & $\begin{array}{l}123 \\
\square \square \square\end{array}$ & $\begin{array}{l}4 \\
\square\end{array}$ & 5 & $\begin{array}{l}123 \\
\square \square \square\end{array}$ & $\begin{array}{l}45 \\
\square \square\end{array}$ & $\begin{array}{l}12 \\
\square \square\end{array}$ & 3 & $\begin{array}{l}4 \\
\square\end{array}$ & 5 \\
\hline 2 & $\begin{array}{lll}1 \square & 2 \square & 3 \square \\
4 \square & 5 \square & 6 \square \\
7 \square & 8 \square & 9 \square\end{array}$ & $\begin{array}{l}123 \\
\square \square \square\end{array}$ & $\begin{array}{l}4 \\
\square\end{array}$ & 5 & $\begin{array}{l}123 \\
\square \square \square\end{array}$ & $\begin{array}{l}4 \quad 5 \\
\square \square\end{array}$ & $\begin{array}{l}12 \\
\square \square\end{array}$ & 3 & $\begin{array}{l}4 \\
\square\end{array}$ & 5 \\
\hline 3 & $\begin{array}{lll}1 \square & 2 \square & 3 \square \\
4 \square & 5 \square & 6 \square \\
7 \square & 8 \square & 9 \square\end{array}$ & $\begin{array}{r}123 \\
\square \square \square\end{array}$ & $\begin{array}{l}4 \\
\square\end{array}$ & 5 & $\begin{array}{r}123 \\
\square \square \square\end{array}$ & $\begin{array}{l}4 \quad 5 \\
\square \square\end{array}$ & $\begin{array}{l}12 \\
\square \square\end{array}$ & 3 & $\begin{array}{l}4 \\
\square\end{array}$ & 5 \\
\hline 4 & $\begin{array}{lll}1 \square & 2 \square & 3 \square \\
4 \square & 5 \square & 6 \square \\
7 \square & 8 \square & 9 \square\end{array}$ & $\begin{array}{l}123 \\
\square \square \square\end{array}$ & $\begin{array}{l}4 \\
\square\end{array}$ & $\begin{array}{l}5 \\
\square\end{array}$ & $\begin{array}{l}123 \\
\square \square \square\end{array}$ & $\begin{array}{l}4 \quad 5 \\
\square \square\end{array}$ & $\begin{array}{l}12 \\
\square \square\end{array}$ & 3 & $\begin{array}{l}4 \\
\square\end{array}$ & 5 \\
\hline 5 & $\begin{array}{lll}1 \square & 2 \square & 3 \square \\
4 \square & 5 \square & 6 \square \\
7 \square & 8 \square & 9 \square\end{array}$ & $\begin{array}{r}123 \\
\square \square \square\end{array}$ & $\begin{array}{l}4 \\
\square\end{array}$ & 5 & $\begin{array}{r}123 \\
\square \square \square\end{array}$ & $\begin{array}{l}45 \\
\square \square\end{array}$ & $\begin{array}{l}12 \\
\square \square\end{array}$ & 3 & $\begin{array}{l}4 \\
\square\end{array}$ & 5 \\
\hline 6 & $\begin{array}{lll}1 \square & 2 \square & 3 \square \\
4 \square & 5 \square & 6 \square \\
7 \square & 8 \square & 9 \square\end{array}$ & $\begin{array}{l}123 \\
\square \square \square\end{array}$ & $\begin{array}{l}4 \\
\square\end{array}$ & 5 & $\begin{array}{l}123 \\
\square \square \square\end{array}$ & $\begin{array}{l}4 \quad 5 \\
\square \square\end{array}$ & $\begin{array}{l}12 \\
\square \square\end{array}$ & 3 & $\begin{array}{l}4 \\
\square\end{array}$ & 5 \\
\hline 7 & $\begin{array}{lll}1 \square & 2 \square & 3 \square \\
4 \square & 5 \square & 6 \square \\
7 \square & 8 \square & 9 \square\end{array}$ & $\begin{array}{r}123 \\
\square \square \square\end{array}$ & $\begin{array}{l}4 \\
\square\end{array}$ & $\begin{array}{l}5 \\
\square\end{array}$ & $\begin{array}{r}123 \\
\square \square \square\end{array}$ & $\begin{array}{l}4 \quad 5 \\
\square \square\end{array}$ & $\begin{array}{l}12 \\
\square \square\end{array}$ & 3 & $\begin{array}{l}4 \\
\square\end{array}$ & 5 \\
\hline $\begin{array}{l}\text { (se anexa } \\
\text { hoja con } \\
\text { más líneas) }\end{array}$ & $\begin{array}{lll}1 \square & 2 \square & 3 \square \\
4 \square & 5 \square & 6 \square \\
7 \square & 8 \square & 9 \square\end{array}$ & $\begin{array}{r}123 \\
\square \square \square\end{array}$ & $\begin{array}{l}4 \\
\square\end{array}$ & $\begin{array}{l}5 \\
\square\end{array}$ & $\begin{array}{r}123 \\
\square \square \square\end{array}$ & $\begin{array}{l}4 \quad 5 \\
\square \square\end{array}$ & $\begin{array}{l}12 \\
\square \square\end{array}$ & 3 & $\begin{array}{l}4 \\
\square\end{array}$ & $\begin{array}{l}5 \\
\square\end{array}$ \\
\hline
\end{tabular}

Fuente: elaboración propia. 
acuícola. Para la identificación de los contactos de las empresas, se empleó en el cuestionario la técnica denominada free recall, que consiste en permitir que el informante traiga libremente a la memoria los nombres de sus fuentes de información, en vez de presentarle una lista de nombres para seleccionar sus contactos de manera preorientada. Esta técnica es muy utilizada en investigaciones realizadas con el enfoque del ARs (Wasserman y Faust, 1994; Knoke y Yang, 2008). La información que proporcionó la encuesta fue clave para evaluar cómo han sido las relaciones en términos de frecuencia de comunicación, cantidad e importancia de la información y el conocimiento que reciben las empresas (recuadro 1).

\section{Aplicación del análisis de redes sociales para la obtención de sociogramas de las redes de flujo de conocimiento e información tecnológica}

La información obtenida de la encuesta se procesó de tal manera que se pudieran aplicar las herramientas de análisis del ARs. En primer lugar, se sistematizó mediante el software Ucinet, con la finalidad de obtener mediante esta herramienta la elaboración de grafos que permiten la "reconstrucción" de la estructura de las redes analizadas. Los lazos interorganizacionales que sostienen las empresas para conseguir información técnica fueron representados mediante los denominados sociogramas, que pueden definirse como gráficos en los cuales las empresas se representan como nodos y las relaciones entre ellos como líneas. Estos diagramas resultan ser muy útiles para descubrir las estructuras ocultas de los grupos por medio de la identificación de protagonistas, alianzas y subgrupos, entre otros.

\section{Estimación de medidas globales de las redes}

Existen varias medidas globales de las redes en el ARs; la mayoría son las mismas empleadas para analizar cualquier otro tipo de red, y las más utilizadas son: a) diámetro; b) distancia media; c) grado medio, y d) densidad.
El diámetro (d max) es la longitud del camino mínimo más largo de la red. En redes grandes, se puede determinar con el algoritmo primero en anchura. Equivale al valor máximo de excentricidad para todos los nodos de la red:

$$
\begin{aligned}
& E(i)=\max _{j \in V \frac{G}{i}} d(i, j) \\
& \max =\max \{E(i): i \in V(G)\}
\end{aligned}
$$

En el contexto del ARs, esta métrica da una idea de la proximidad entre pares de actores en la red e indica cómo están en el peor de los casos. Las redes más dispersas suelen tener un mayor diámetro que las más densas, al existir menos caminos entre cada par de nodos.

La distancia media (d) es una medida que da una idea de qué tan lejos están los distintos actores en promedio. La distancia en el ARS representa la eficiencia del flujo de información en la red:

$$
(d)=\frac{1}{2 \operatorname{Lmax}} \sum_{i, j \neq i} d_{\mathrm{i}} j
$$

$d_{\mathrm{ij}}$ es la distancia geodésica entre los nodos $i$ y $j$

El grado medio (k) es el número promedio de contactos que posee una red, y su cálculo es así:

$$
(k)=\frac{1}{N} \sum_{i=1}^{N} K i
$$

$\mathrm{N}$ = número de nodos de la red

$\mathrm{L}=$ número de enlaces de la red

Por último, la densidad mide el grado de conectividad de la red social a nivel global, y se calcula mediante el siguiente cociente: núm. de relaciones existentes/ núm. de relaciones posibles

\section{Estimación de medidas locales de posición y naturaleza de las relaciones}

La posición de las empresas de la red se estimó con base en el indicadorcentralidad de grado (degreecentrality). El índice de centralidad de grado se calculó mediante el conteo del número de relaciones directas que tiene cada empresa. 
Como puede observarse, el cuestionario aplicado dio la oportunidad a los informantes de evaluar las relaciones que establecen con sus fuentes de información técnica (columnas A, B y C del recuadro 1). Las respuestas fueron recolectadas en una escala Likert de cinco puntos y los registros de cada cuestionario fueron promediados para obtener un indicador de la fuerza de los vínculos para cada empresa encuestada. La diversidad de las relaciones se estimó de acuerdo con lo declarado por las empresas acerca de sus contactos, y estos fueron identificados por tipo de organización en la columna referente al "giro" de los contactos (proveedores, consultores, instituciones de educación superior [IES], Gobierno, etc.). Una empresa tiene relaciones diversas, teniendo en cuenta que sus contactos pertenecen a cada uno de estos sectores de forma acumulativa, es decir, el indicador tiene un mínimo de uno y un máximo de seis.

\section{Resultados}

\section{Características de las empresas encuestadas para el estudio}

La encuesta fue aplicada a una muestra de 91 empresas dedicadas al cultivo de camarón en las dos regiones de estudio. En una primera etapa de la investigación, durante el segundo semestre de 2015, se hizo la encuesta a 33 empresas ubicadas en la zona centro y sur del Estado de Sonora, pertenecientes en su mayoría al municipio de Hermosillo. Posteriormente, durante el primer semestre de 2016 se llevó a cabo la encuesta a 58 plantas acuícolas localizadas en el municipio de Ahome, Sinaloa. De acuerdo con el tamaño de las granjas acuícolas encuestadas, la mayor parte de la muestra estuvo compuesta por unidades de tamaño micro y pequeño (57\% en Sonora y $64 \%$ en Sinaloa). En lo que respecta a la edad de las empresas, se observó que casi la mitad de estas $(48,3 \%)$ son granjas que tienen una antigüedad máxima de 10 años, pero las empresas de 11 a 15 años también representaron una parte importante de la muestra (37,9\%); en tanto que las empresas de 16 a 20 años y las mayores de 20 representaron el 6,9\% y 5,9\%, respectivamente. Por otra parte, la mayoría de las empresas de esta muestra corresponden al sector privado $(82,8 \%)$ y solo un $17,2 \%$ al sector social.

\section{Características globales de las redes de flujo de conocimiento e información tecnológica}

La composición, el tamaño y la densidad de la red, así como el nivel de centralización, son aspectos importantes por considerar cuando se discute el impacto potencial de las redes en la innovación tecnológica, en la difusión de nuevas tecnologías y en la transferencia y el intercambio de conocimiento.

\section{Tamaño y composición de las redes}

Como ya se ha dicho, una estructura de relaciones o una red interorganizacional es el entramado de vínculos establecidos entre los elementos constitutivos de una estructura definida; para el caso concreto, dichos elementos son organizaciones productoras de camarón de cultivo o entidades que poseen recursos para apoyar esta actividad.

La estructura de relaciones interorganizacionales que implican el flujo de conocimiento e información tecnológica en la actividad del cultivo de camarón en la región de Ahome, Sinaloa, está conformada por 115 nodos; de ellos, 58 corresponden a las empresas acuícolas encuestadas y 57, a organizaciones o actores que fueron identificados por las empresas participantes como proveedores de conocimiento e información técnica. La composición de las fuentes de conocimiento e información tecnológica en este caso es la siguiente: 5 empresas competidoras; 4 organismos gubernamentales; 13 IEs y centros de investigación públicos (CIP); 23 proveedores; 10 consultores y 2 organismos de la sociedad civil (tabla 1). 
Tabla 1. Composición de la RFCIT en el cultivo de camarón de la región de Ahome, Sinaloa (centralidad de grado)

\begin{tabular}{|c|c|}
\hline Empresas (58) & \\
\hline Acuacultores Unidos de San Martín S. A. de C. V. & 14 \\
\hline Acuacultores del Quinto Día (Bachomobampo) & 14 \\
\hline Acuacultores del Quinto Día, SPR de RL & 13 \\
\hline Acuacultores del Quinto Día, SPR de RI (Chicura). & 13 \\
\hline SPR de RI Laguna de Oro & 7 \\
\hline Laguna de Oro, sección III & 7 \\
\hline SPR de RI Laguna de Oro, sección IV & 7 \\
\hline Conquistadores Acuícolas, S. A. de C. V. & 7 \\
\hline Acuícola Malinalli, S. A. de C. V. & 7 \\
\hline Azteca Shirmp, S. A. de C. V. & 7 \\
\hline Camarones El Renacimiento, SPR de RI & 7 \\
\hline Ac Márquez & 7 \\
\hline Laguna de Oro, sección II & 6 \\
\hline SPR de RI Laguna de Oro, sección V & 6 \\
\hline Acuícola Santa Rita, & 6 \\
\hline Ac Abulón, SPR de RI & 6 \\
\hline Acuícola Pleamar & 5 \\
\hline sCPA Playa Escondida, SPR de RI & 5 \\
\hline Granja 11 de diciembre, S. A. de C. V. & 5 \\
\hline Ecocampos Acuícola, S. A. de C. V. & 5 \\
\hline Acuícola Roja, SPR de RI & 4 \\
\hline Acuícola Camacoa, SPR de RI & 4 \\
\hline Grupo Acuícola Santa Fe, S. A. de C. V. & 4 \\
\hline Alba Nidia Moreno Cruz (Sangialjaruri) & 4 \\
\hline scPp Acuícola Camarón Norte, SCL & 4 \\
\hline scPp Campos Acuícola, sc de RI & 4 \\
\hline $\begin{array}{l}\text { Acuícola Camargo, S. A. de C.V. } \\
\text { (Los Sánchez Cota, sector II) }\end{array}$ & 4 \\
\hline scPp y de Acuacultores, Los Sánchez Cota, sc de RL & 4 \\
\hline Acuacultura Matacahui & 4 \\
\hline Playa Las Piedras Acuícolas & 4 \\
\hline AC Bioplanet & 3 \\
\hline Acuícola 11 diciembre & 3 \\
\hline Aqua Consult, S. A. de C. V. & 3 \\
\hline
\end{tabular}

\begin{tabular}{|c|c|}
\hline AC Santo Niño & 3 \\
\hline Acuícola Camargo, S. A. de C. V. & 2 \\
\hline Las Pichihuilas, S. A. de C. V. & 2 \\
\hline Ecoshirmp Supply, S. A de C. V & 2 \\
\hline Acuícola Baja Mar, S. A. de C. V. & 2 \\
\hline Acuícola Rotcher, S. A. de C. V & 2 \\
\hline Acuícola Tierrad de Canaan, S. A. de C. V. & 2 \\
\hline Acuícola Camargo, S. A. de C. V. (General Leyva) & 2 \\
\hline scpp Edulfo González, sc de RL & 2 \\
\hline Ac Villa Oceánica & 2 \\
\hline Nueva Higuera, SPR de RL & 2 \\
\hline Playa Tadeo & 2 \\
\hline Los Sánchez Cota, sc de RL & 2 \\
\hline El Bichi, Estero SPR de RL & 2 \\
\hline Granja AC Ricardo & 2 \\
\hline SCPA A.G.S. CV de RL & 2 \\
\hline JM Acuacultura S.A. de C. V. & 1 \\
\hline Acuícola Las Gaviotas, SPR de RL & 1 \\
\hline A.C. San Ricardo & 1 \\
\hline A.C. Los Mochis & 1 \\
\hline A.C. Marion, S. A. de C. V. & 1 \\
\hline Playa Baja, SPR de RL & 1 \\
\hline Granja A. C. Estuario de Río Fuerte sc de RL & 1 \\
\hline San Isidro, SPR de RL & 1 \\
\hline Granja AC 14 & 1 \\
\hline Competidores (5) & \\
\hline
\end{tabular}

Planta Solman 16

A. C. Rotcher 7

Productos Pesqueros del Evora 4

A. C. Bioplanet 1

Camarones orgánicos 1

Organismos gubernamentales (4)

\begin{tabular}{lc} 
CESASIN & $\frac{49}{12}$ \\
\hline Conapesca & $\frac{1}{7}$ \\
\hline Sagarpa & 4 \\
\hline INADEM & Continúa
\end{tabular}


Continuación Tabla 1. Composición de la RFCIT en el cultivo de camarón de la región de Ahome, Sinaloa (centralidad de grado)

\begin{tabular}{|c|c|}
\hline \multicolumn{2}{|l|}{ IES y centros de investigación (13) } \\
\hline CIAD Mazatlán & 16 \\
\hline U. de Occidente (UDO) & 12 \\
\hline CIBNOR - Sonora & 8 \\
\hline CIIDIR/IPN & 7 \\
\hline ICMYL/UNAM & 4 \\
\hline TEC. MAR. LAB. & 4 \\
\hline Instituto Tecnológico de Sonora (ITSON) & 4 \\
\hline CIAD - Culiacán & 1 \\
\hline CIAD - Guaymas & 1 \\
\hline Laboratorio de alimentos & 1 \\
\hline Universidad Autónoma de Sinaloa (UAS) & 1 \\
\hline Tecnológico de Monterrey (TEC) & 1 \\
\hline \multicolumn{2}{|l|}{ Proveedores (23) } \\
\hline Agribands Purina (Obregón) & 7 \\
\hline Provimi Obregón & 6 \\
\hline Proveedor de larvas (Mazatlán) & 5 \\
\hline Fitmar & 5 \\
\hline Gene Reach Biotchnology Corp. (USA) & 4 \\
\hline Innovaciones Acuícolas & 4 \\
\hline Aquapacific & 4 \\
\hline Vimifos & 2 \\
\hline Lab. Alimentos & 2 \\
\hline Rev. Divulgación Industrial AC & 2 \\
\hline Ab. Comb & 1 \\
\hline Bioplanet & 1 \\
\hline
\end{tabular}

\begin{tabular}{|c|c|}
\hline BFI Innovation & 1 \\
\hline Innova Acuícola (Culiacán) & 1 \\
\hline Yorba A. Camarón & 1 \\
\hline Lab. A. Químicos & 1 \\
\hline Grumar (Cruz de Elota, Sinaloa) & 1 \\
\hline Ferias nacionales & 1 \\
\hline Ferias internacionales & 1 \\
\hline Agua Marina & 1 \\
\hline 0. Proveedores & 1 \\
\hline Selectos del Mar & 1 \\
\hline Rev. Acuícolas & 1 \\
\hline \multicolumn{2}{|l|}{ Consultoría (10) } \\
\hline One Lab & 4 \\
\hline Concepto Azul & 4 \\
\hline SLA (Redes, Yahoo) Shirmp News & 4 \\
\hline Biol. Donald Liner & 3 \\
\hline Clound Boy (calidad de agua, USA) & 3 \\
\hline Biol. Jairo Sarmiento & 3 \\
\hline Asi Es (consultora empresarial) & 1 \\
\hline Biol. Jesús Meléndez (Ahome) & 1 \\
\hline Biol. Humberto Robles & 1 \\
\hline Biol. D. Flores & 1 \\
\hline \multicolumn{2}{|l|}{ Asociaciones civiles (2) } \\
\hline Acuacultores de Ahome A.C. & 25 \\
\hline Clúster Sinaloa & 1 \\
\hline
\end{tabular}

Fuente: elaboración propia.

Las empresas cultivadoras de camarón en la región de Ahome, Sinaloa, identificaron como principales proveedores de información de nuevas tecnologías, productos e información técnica a las empresas proveedoras de alimentos balanceados para el camarón; por ejemplo, Agribands Purina, Provimi-Cargill, vimifos, AquaMarina, Innovaciones Acuícolas S. A., varias de ellas ubicadas en Obregón, Sonora. También ocupan un lugar destacado en este aspecto las empresas proveedoras de

larvas de camarón, como AquaPacific, FIMAR S. A. Concepto Azul (Ecuador) y GeneReach Biotechnology Corp., ubicada en Estados Unidos, que es una importante proveedora de equipos médicos, medicamentos, reactivos y consumibles para análisis, y servicios de asesoría técnica en salud animal. Son también importantes los proveedores de probióticos y complementos nutricionales, como la empresa de biotecnología Bioplanet, situada en Culiacán, Sinaloa. Los consultores resultan ser 
otro proveedor relevante de conocimiento para la actividad acuícola desarrollada en el norte de Sinaloa. Se destaca, por ejemplo, la empresa OneLab, que es una plataforma de e-commerce de Argentina utilizada por los productores de camarón para comprar y vender equipos, insumos y servicios de laboratorio. Otro consultor relevante en esta red es la empresa ecuatoriana Concepto Azul S.A, especializada en la biotecnología acuícola, agrícola y ambiental. Esta empresa cuenta con alrededor de 50 profesionales y técnicos con diferentes niveles de especialización (doctores, masters, biólogos, acuicultores, tecnólogos) en genética, patología, inmunología, microbiología, fisiología, biología molecular e ingeniería genética. Tiene, además, grandes bases de información, bibliografía y documentación científica actualizada en los diferentes ámbitos de su actividad (cultivos de camarón, piscicultura, agricultura, agroforestal, biodiversidad). Las universidades y los centros de investigación también juegan un papel muy importante, en cuanto fuentes de conocimiento para las empresas cultivadoras de camarón. Se destacan, en primer lugar, por su nivel de centralidad en la red el Centro de Investigación en Alimentación y Desarrollo (CIAD), sede Mazatlán, y la Universidad de Occidente, con sedes en Guasave, Culiacán y Mazatlán, Sinaloa. En segundo lugar, están instituciones como el Instituto pedagógico nacional (IPN) y el Centro de Investigaciones Biológicas del Noroeste (CIBNOR). A diferencia de Sonora, en Sinaloa las organizaciones de la sociedad civil juegan un papel central en la red de conocimiento de la acuicultura. Destaca la Asociación de Acuacultores de Ahome, AC, como se ha señalado anteriormente (tabla 1).

En el caso de Sonora, las RFCIT de las actividades de cultivo de camarón desarrolladas en la región centro-sur están compuestas por 81 nodos; de estos, 33 son las organizaciones que componen la muestra seleccionada de plantas acuícolas para el estudio, es decir, las organizaciones a partir de las cuales se obtuvieron los datos relacionales. Las empresas acuícolas sonorenses participantes en la encuesta identificaron 48 organizaciones de distinto tipo que les proveen información y asesoramiento en cuestiones técnicas, y que coadyuvan a la puesta al día en nuevas tecnologías de dicho campo.
Este número es ligeramente inferior al que arrojó el caso de Sinaloa, donde las empresas identificaron un total de 57 organizaciones que funcionan como fuentes externas de conocimiento e información tecnológica. De los 48 elementos o nodos que son fuentes de los flujos de conocimiento e información en el caso de Sonora, 13 son instituciones de educación superior o centros públicos de investigación; 8 son empresas dedicadas a la consultoría técnica en el ramo acuícola; 9 son organismos pertenecientes a distintos niveles de gobierno; 16 son proveedores de las plantas cultivadoras de camarón, y 2 son organizaciones de la sociedad civil (tabla 2).

En el caso de la región centro-sur de Sonora, en el grupo de 13 IES y los CIP, juegan un papel central el Centro de Investigaciones Biológicas del Noroeste (CIBNOR), con sedes en La Paz, BC, Hermosillo y Guaymas, Sonora; el Instituto Tecnológico de Sonora (ITSON); el CIAD y la Universidad de Sonora (Unison) (tabla 3). De los consultores que integran parte de la red Sonora, los más relevantes pertenecen a empresas especializadas en la consultoría en cuestiones ambientales; por ejemplo, la empresa GAPSA es especializada en asesoría jurídica y en manejo ambiental. Los organismos gubernamentales identificados como fuentes de información ascendieron a nueve en el caso de Sonora; entre ellos se encuentra el nodo central de la red, la Comisión de Sanidad Acuícola del Estado de Sonora (Cosaes), y sobresalen también Senasica y Sagarpa en este rubro. Entre los proveedores de las empresas acuícolas que constituyen fuertes proveedores de conocimiento técnico, se destacan las empresas proveedoras de alimentos y complementos nutricionales (Purina, Vimifos, Provimi, Zeigler) y las proveedoras de larvas de camarón (AquaPacific S. A., por ejemplo). Por último, las organizaciones de la sociedad civil juegan un papel marginal o periférico en la red de conocimiento de la acuicultura sonorense (tabla 2).

Un aspecto relevante en este análisis es la naturaleza de las relaciones que contiene cada una de las redes; en ambas redes las relaciones imperantes son de tipo interorganizacional en sentido estricto, es decir, integradas por nodos heterogéneos, de acuerdo con el sector de origen (privado, público, social, académico). 
Tabla 2. Composición de la RFCIT en el cultivo de camarón de la región centro-sur de Sonora (centralidad de grado)

\begin{tabular}{|c|c|c|c|c|c|}
\hline \multicolumn{2}{|l|}{ Empresas (33) } & \multicolumn{2}{|l|}{ IES y centros de investigación (13) } & \multicolumn{2}{|l|}{ Proveedores (16) } \\
\hline Maricultura del Pacífico & 13 & Instituto Tecnológico de Son. & 9 & Vimifos & 8 \\
\hline La Borbolla & 10 & CIAD - Hermosillo & 9 & Purina & 6 \\
\hline Gran Kino Sinaloense & 8 & CIBNOR - Hermosillo & 8 & Aquapacific & 3 \\
\hline Acuícola La Tatahuila & 8 & Universidad de Sonora & 7 & Cargill & 3 \\
\hline Río Azul & 8 & CIBNOR - La Paz B.C.S. & 5 & Zeigler & 3 \\
\hline Kiwis Acuicultores & 7 & Dictus -Universidad de Sonora & 3 & Nutrimar & 3 \\
\hline Larvas Génesis & 7 & Universidad de Arizona & 3 & Silver Cap & 1 \\
\hline Acuícola San Fernando & 6 & CIBNOR - Guaymas & 2 & Alibio & 1 \\
\hline Acuícola Oasis & 6 & Universidad de Auburn & 1 & Kishmar & 1 \\
\hline Ojai & 6 & Universidad Autónoma de Morelos & 1 & Malta Clayton & 1 \\
\hline Granjas Marinas de Sonora S. A. de C. V. & 6 & CIAD - Mazatlán & 1 & Ecobiosmar & 1 \\
\hline 11 de diciembre & 6 & CICESE & 1 & Proaqua & 1 \\
\hline La Piedreña & 6 & Universidad Estatal de Sonora & 1 & Genitec & 1 \\
\hline Llaos Acuacultura, S. A. de C. V & 5 & Consultoría (8) & & Aquamar & \\
\hline Cumora & 4 & GAPSA & 7 & Provimi & 1 \\
\hline Camarón Dorado & 4 & Oswaldo Serna & 3 & Insumos A.C. & 1 \\
\hline Acuaproductos Fico & 4 & Gabriel Hernández & 2 & Asociaciones civiles (2) & \\
\hline Gral. Ignacio Pesqueira & 4 & Asesoría en producción & 1 & Asoc. de Acuicultores de Sonora & 1 \\
\hline Acuícola Sayri & 3 & CIA Alimentos de Animales S. A. & 1 & ANPLAC & 1 \\
\hline Acuícola Soid & 3 & NSF International & 1 & & \\
\hline Acuícola San Carlos & 3 & Asesoría y Análisis de Guaymas & 1 & & \\
\hline Quinta San Fabián & 3 & Ernesto Garmendia & 1 & & \\
\hline Santa Rosalía & 3 & Organismos gubernamentales (8) & & & \\
\hline Acuícola P\&P S. A. de CV & 3 & Cosaes & 31 & & \\
\hline Pmg Acuícola Sol y Mar S. A. de CV & 3 & Sagarpa & 7 & & \\
\hline Santa Magda & 3 & Senasica & 5 & & \\
\hline Selecta & 3 & Instituto de Acuacultura & 3 & & \\
\hline Aquactiva & 3 & Subsecretaría de Pesca & 2 & & \\
\hline Aquacultivos de Kino & 3 & Subdelegación Conapesca & 1 & & \\
\hline Acuícola Alcatraz & 3 & SEMARNAT & 1 & & \\
\hline Doña Kika & 3 & PROFEPA & 1 & & \\
\hline Guaymar Acuícola S. A. de CV & 2 & Senasica & 1 & & \\
\hline Engocam & 1 & & & & \\
\hline
\end{tabular}

Fuente: elaboración propia. 
Sin embargo, mientras que en la red de Sonora el $100 \%$ de las relaciones identificadas son de carácter interorganizacional (ninguna empresa de esta entidad mencionó a otra empresa como fuente de conocimiento), en el caso de la red de Sinaloa sí están presentes las relaciones interempresariales que implican flujos de conocimiento, ya que cinco de ellas declararon que uno de sus proveedores de conocimiento técnico era otra empresa acuícola.

\section{Tamaño, grado medio, distancia y densidad de las redes de flujo de conocimiento e información tecnológica}

En relación con el tamaño y las características generales de las redes analizadas, se tiene que al número de nodos de la red de Sonora le corresponde un número de relaciones potenciales igual a 6400, pero las relaciones que efectivamente se presentan ascienden solo a 316, lo que resulta en una densidad de la red igual a 0,049; esto quiere decir que de las relaciones que potencialmente se pueden alcanzar, solo se establece el 4,9\%. En lo que respecta a Sinaloa, el número de relaciones posibles es 13,225, pero se registran 493 relaciones efectivas, lo que arroja un índice de densidad de la red igual a 0,038 (tabla 3). Este indicador debe ser tomado con precaución, ya que el número de relaciones posibles de una red crece exponencialmente cuando es mayor el número de nodos integrantes. Si se considera el número de relaciones realmente presentes en cada una de las redes, es posible observar que es mucho mayor el correspondiente a Sinaloa. Las figuras 2 y 3 permiten apreciar una mayor densidad y conectividad en la red de Sinaloa con respecto a la de Sonora. El indicador de grado medio es también un indicador de la densidad y conectividad de las redes, siendo el de la red Sinaloa superior al de la red Sonora (tabla 3).

Otro indicado del tamaño de la red es el diámetro, que representa la distancia geodésica más larga para llegar de un nodo a otro en la red. En el caso de la red Sonora el diámetro es igual a seis nodos, mientras que para Sinaloa es igual a siete. La distancia también es un buen indicador del tamaño de la red, porque indica el número de nodos que las empresas alcanzan en promedio. La distancia promedio de la red Sonora es de 3,006 y la de Sinaloa es de 3,092.

Tabla 3. Características de la estructura de la RFCIT del cultivo de camarón de Sinaloa y Sonora

\section{Sinaloa Sonora}

\begin{tabular}{lll}
\hline Tamaño (N. ${ }^{\circ}$ de nodos) & 114 & 81 \\
\hline Diámetro & 7 & 6 \\
\hline Distancia & 3,092 & 3,006 \\
\hline Densidad & 0,038 & 0,049 \\
\hline Grado medio & 4,298 & 3,901 \\
\hline Desv. estándar & 1,055 & 0,922 \\
\hline
\end{tabular}

Fuente: elaboración propia.

\section{Topología o arquitectura de las redes}

El análisis visual de los grafos resultantes en el ejercicio de reconstrucción de las RFCIT de las dos regiones de estudio (figuras 2 y 3 ) permite observar similitudes entre la forma general que tienen estas dos redes; en ambos casos se observa una estructura tipo estrella. Una red en estrella es una red donde los nodos están conectados principal y directamente a un punto central, y todas las comunicaciones se hacen a través de ese punto (el punto concentrador de las emisiones de información).

\section{Nivel de centralización}

Partiendo de la representación gráfica de las redes (figuras 2 y 3), una característica que se observa es que se trata de dos redes altamente centralizadas; sin embargo, el grado de centralización es mayor en el caso de la red correspondiente al caso Sonora, que es una red totalmente centralizada donde todos los nodos, menos uno, son periféricos y solo pueden comunicarse a través del nodo central. Se puede ver en los grafos que la red de Sonora es más centralizada que la de Sinaloa. En el primer caso destaca la importancia y centralidad que tiene un único nodo de la red: la Cosaes, mientras que un importante número de nodos 
tienen un carácter estrictamente periférico ( 35 de un total de 81 nodos). En cambio, la red de Sinaloa muestra la centralidad y relevancia que tienen como emisores de información técnica más de un nodo. En este caso, aparte de la Comisión Estatal de Sanidad, se encuentra una organización de la sociedad civil encargada de promover la actualización técnica, la capacitación y profesionalización de los productores, la difusión de los adelantes tecnológicos en nutrición y sanidad animal. Se trata de la asociación Acuacultores de Ahome, AC. Es relevante, asimismo, el caso de la red de Sinaloa, donde el entramado de conexiones que implican la transferencia de conocimiento se hace más denso en la parte derecha del grafo alrededor de cuatro empresas productoras que poseen una posición preponderante en la red, fenómeno que no se da en la red de Sonora.

Una de las desventajas de las redes excesivamente centralizadas es la dependencia de la red en el comportamiento y la eficiencia de un nodo, que por lo general funciona como el emisor preponderante de la información o tiene el control de la red. Al fallar este nodo, o ser ineficiente, se compromete el funcionamiento y la eficiencia de la red en su conjunto. Este tipo de redes confieren un poder muy grande al nodo protagónico.

El nivel de centralización de una red tiene implicaciones importantes en términos de aprendizaje tecnológico, de asimilación de nuevo conocimiento y tecnologías, adopción y difusión de las innovaciones tecnológicas, ya que influye, por ejemplo, en cuestiones como hasta qué punto otros actores pueden participar en la red, el poder de negociación de los diferentes actores o el control de la información (Nooteboom, 2004). Pietrobelli y Rabellotti (2009) muestran que las formas muy centralizadas de redes con patrones jerárquicos o en cautividad de interacción tienden a obstaculizar la transferencia de conocimientos y el aprendizaje interactivo y la innovación, por lo tanto, es menos probable que estos procesos ocurran. En redes más descentralizadas, donde no hay un actor dominante claro que controla lazos directos importantes, otros actores pueden acceder a la información de manera más fluida.

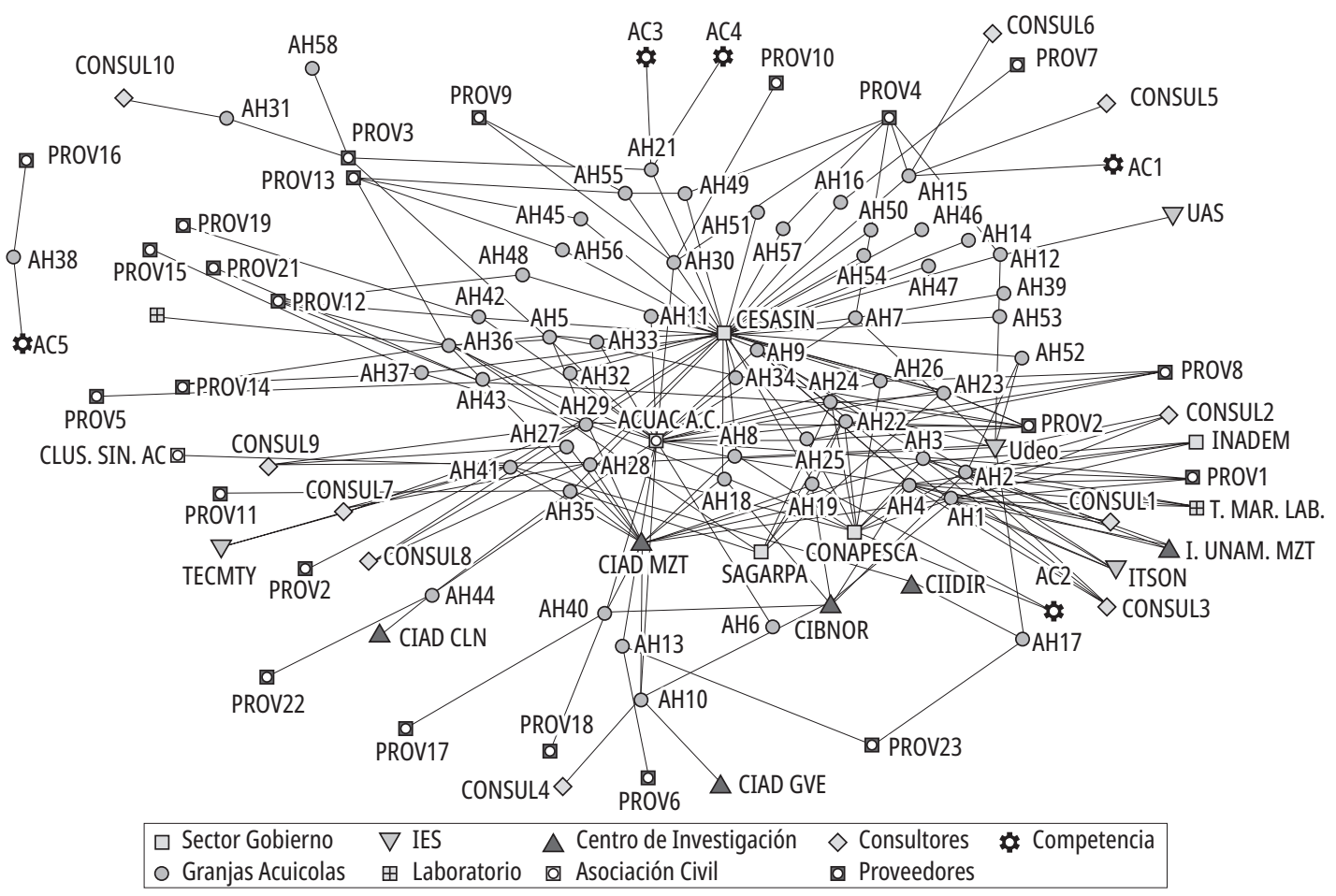

Figura 2. Grafo de la RFCIT de las plantas de cultivo de camarón del municipio de Ahome, Sinaloa Fuente: elaboración propia mediante NETDraw 2.138. 


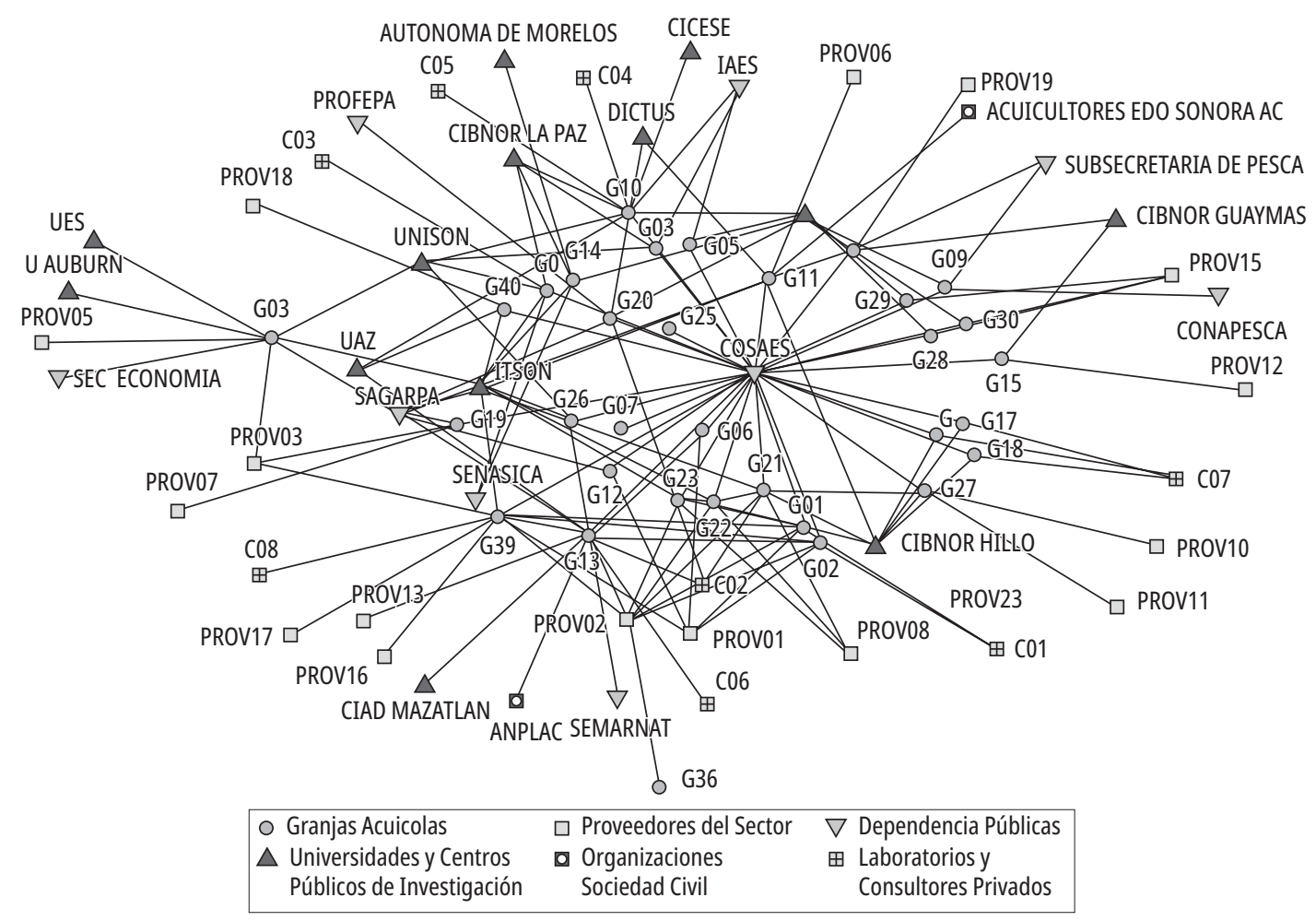

Figura 3. Grafo de la RFCIT de las plantas de cultivo de camarón en la región centro-sur del Estado de Sonora Fuente: elaboración propia.

Un aumento en el grado de centralidad podría implicar un riesgo si el actor central restringe el acceso a la información a otros actores, pero también podría facilitar la coordinación de los diferentes actores que pudieran ser necesarias en procesos de innovación complejos. Acerca de la relación entre la centralidad de la red y la innovación, Ibarra (1993) sostiene que la centralidad de la red es el determinante más fuerte de la participación individual en los esfuerzos para la innovación administrativa, pero no para la innovación tecnológica.

\section{Alcance territorial de las redes}

En este aspecto existen más similitudes que diferencias entre las dos redes analizadas, ya que ambas exhiben la existencia de nodos que se encuentran fuera de su territorio regional, incluso algunos ubicados en el extranjero. Se trata entonces de redes con cierto grado de apertura geográfica por sus alcances territoriales. En ambas redes el entramado de relaciones que implican flujos de conocimiento se extiende más allá de sus contextos cercanos y está abierto a la incorporación de actores y organizaciones alejadas. En el caso de Sonora, hay nexos de los productores son dos universidades de Estados Unidos y con varios proveedores norteamericanos y sudamericanos, en especial cuando se trata de proveedores de equipos y reactivos clínicos y de medicamentos, probióticos y antibióticos avanzados. En el caso de Sinaloa, cuatro de los diez consultores se ubican en el extranjero. Asimismo, dentro del conjunto de proveedores, cuatro son organizaciones o agentes ubicados en el extranjero y varios en Sonora, principalmente en Obregón.

Lo anterior permite definir que el entorno de la organización no queda determinado por el territorio al cual hace parte, es decir, local, estatal o nacional, teniendo en cuenta que para el caso concreto, el sector internacional pertenece al entorno de algunas organizaciones. En concreto, se quiere dejar claro que el medio ambiente de las organizaciones no es el territorio donde actúa, sino más bien las relaciones que establecen estas 
y la estructura a la cual hacen parte. No obstante, esto no implica que la estructura no pueda ser delimitada, pues la delimitación es necesaria para poder estudiar redes, debido a que su magnitud puede ser una gran dificultad para entender las dinámicas entre elementos especificados (Brand et al., 2007).

\section{Posicionamiento de los actores individuales/nivel de centralidad}

El posicionamiento de los actores individuales y la manera como están conectados a otros actores de la red tienen también importantes implicaciones en términos de innovación y adopción tecnológica. Un posicionamiento central, tener una posición como intermediario en la red o contar con un vínculo estrecho con un actor que tiene múltiples conexiones son todas posiciones que garantiza el acceso a los recursos críticos (Wang et al., 2015; Nooteboom, 2004). Por el contrario, una posición periférica en la red y la necesidad de ir a través de muchos nodos para acceder a la información relevante son condiciones que podrían obstaculizar gravemente el flujo de conocimiento dentro de la red y, por lo tanto, la innovación. En suma, los actores con una posición "más central" tienen un acceso más fácil y rápido a los demás actores de la red (útil para acceder a recursos como información) y una mayor capacidad para ejercer un control del flujo entre ellos.

Como se ha indicado anteriormente, se estimó una medida de centralidad (de grado) para todos y cada uno de los nodos integrantes de las dos redes analizadas. Los resultados pueden observarse en las tablas 1 y 2 . La centralidad de grado fue propuesta en el trabajo seminal de Freeman (1979) para redes no ponderadas; esta centralidad es la primera y más simple de las medidas de centralidad, y corresponde al número de enlaces que posee un nodo con los demás. Ya se ha señalado que en promedio la red de Sinaloa tiene una media de centralidad de grado superior a la red Sonora $(4,298$ y 3,901, respectivamente), y también se ha señalado que en caso de Sonora la centralidad de grado más alta corresponde al Cosaes (31) y en Sinaloa a Cosasin (49) y Acuicultores de Ahome (25).

\section{Características de las relaciones empresas-proveedores de conocimiento}

\section{La fuerza de los lazos plantas acuícolas-proveedores de conocimiento}

Como ha sido indicado en el apartado que describe el método del estudio, la encuesta llevada a cabo incluyó un reactivo para evaluar la fortaleza/debilidad de las relaciones que establecen las empresas acuícolas con sus fuentes de información técnica (recuadro 1). Los registros de cada cuestionario fueron ponderados para obtener un indicador de la fuerza de los vínculos para cada empresa encuestada. Los resultados se muestran en la tabla 4.

La literatura sobre la fuerza de los vínculos se refiere principalmente a la naturaleza del vínculo relacional entre dos o más actores sociales, así como al efecto de este vínculo en sus actividades de intercambio de información (Rost, 2011; Hansen et al., 2005). Quienes han estudiado el tema, por lo general, clasifican la relación entre los actores sociales como enlazados por un vínculo fuerte o uno débil. Se considera que los lazos fuertes tienen mayores niveles de cercanía, reciprocidad y compromiso que los lazos débiles (Rost, 2011). Aunque existe un debate considerable acerca de las ventajas relativas de estos dos tipos de vínculos, es aceptado que los lazos fuertes aumentan la probabilidad de que los actores sociales compartan información sensible entre ellos, mientras que los lazos débiles brindan acceso a una mayor cantidad y diversidad de información (Hansen, 1999). Las tablas 4 y 5 contienen los resultados en cuanto a la fuerza de los vínculos de las empresas acuícolas con su red de proveedores de conocimiento y tecnologías.

Como puede apreciarse en las tablas 4 y 5, en comparación con lo que sucede en Sonora, la red de transferencia de conocimiento de Sinaloa tiene una mayor proporción de productores acuícolas que mantiene lazos muy fuertes con las organizaciones que constituyen su red de proveedores de conocimiento técnico: el $62,1 \%$ contra un 51,1 en Sonora. 
Tabla 4. Fuerza de los lazos (tie strength) en la RFCiT en el cultivo de camarón: Sinaloa versus Sonora

\begin{tabular}{|c|c|c|c|c|c|c|c|}
\hline \multicolumn{4}{|c|}{ Sinaloa } & \multicolumn{4}{|c|}{ Sonora } \\
\hline Nodo & $\begin{array}{l}\text { índice de fuerza } \\
\text { de los lazos }\end{array}$ & Nodo & $\begin{array}{l}\text { indice de fuerza } \\
\text { de los lazos }\end{array}$ & Nodo & $\begin{array}{l}\text { índice de fuerza } \\
\text { de los lazos }\end{array}$ & Nodo & $\begin{array}{l}\text { índice de fuerza } \\
\text { de los lazos }\end{array}$ \\
\hline 01 & 3,5 & 30 & 4,0 & 01 & 3,33 & 30 & 0,83 \\
\hline 02 & 3,7 & 31 & 4,0 & 02 & 3,33 & 36 & 3,33 \\
\hline 03 & 3,8 & 32 & 3,5 & 03 & 2,50 & 39 & 2,50 \\
\hline 04 & 3,8 & 33 & 4,0 & 04 & 1,67 & 40 & 5,00 \\
\hline 05 & 5,0 & 34 & 5,0 & 05 & 1,67 & & \\
\hline 06 & 5,0 & 35 & 5,0 & 06 & 1,67 & & \\
\hline 07 & 4,5 & 36 & 4,5 & 07 & 1,67 & & \\
\hline 08 & 5,0 & 37 & 5,0 & 08 & 5,00 & & \\
\hline 09 & 3,5 & 38 & 4,5 & 09 & 0,83 & & \\
\hline 10 & 4,0 & 39 & 5,0 & 10 & 5,00 & & \\
\hline 11 & 5,0 & 40 & 2,8 & 11 & 0,83 & & \\
\hline 12 & 5,0 & 41 & 3,4 & 12 & 4,17 & & \\
\hline 13 & 3,7 & 42 & 4,8 & 13 & 2,50 & & \\
\hline 14 & 5,0 & 43 & 4,5 & 14 & 5,00 & & \\
\hline 15 & 5,0 & 44 & 4,5 & 15 & 3,33 & & \\
\hline 16 & 3,5 & 45 & 5,0 & 16 & 4,17 & & \\
\hline 17 & 3,7 & 46 & 5,0 & 17 & 4,17 & & \\
\hline 18 & 5,0 & 47 & 4,0 & 18 & 4,17 & & \\
\hline 19 & 5,0 & 48 & 5,0 & 19 & 3,33 & & \\
\hline 20 & 4,4 & 49 & 5,0 & 20 & 2,50 & & \\
\hline 21 & 4,4 & 50 & 5,0 & 21 & 3,33 & & \\
\hline 22 & 4,7 & 51 & 5,0 & 22 & 4,17 & & \\
\hline 23 & 4,5 & 52 & 3,7 & 23 & 4,17 & & \\
\hline 24 & 4,5 & 53 & 4,5 & 24 & 2,50 & & \\
\hline 25 & 4,5 & 54 & 5,0 & 25 & 2,50 & & \\
\hline 26 & 4,5 & 55 & 4,5 & 26 & 2,50 & & \\
\hline 27 & 3,0 & 56 & 4,5 & 27 & 5,00 & & \\
\hline 28 & 3,0 & 57 & 4,5 & 28 & 0,83 & & \\
\hline 29 & 3,0 & 58 & 5,0 & 29 & 0,83 & & \\
\hline
\end{tabular}

Fuente: elaboración propia.

Tabla 5. Cuadro comparativo resumen de la fuerza de las relaciones en la RFCIT de las empresas acuícolas: Sinaloa versus Sonora

\begin{tabular}{l|c|c|l|c|c}
\hline $\begin{array}{l}\text { Fuerza de las relaciones } \\
\text { (tie strength) }\end{array}$ & $\begin{array}{c}\text { N. }{ }^{\circ} \text { de } \\
\text { empresas }\end{array}$ & $\begin{array}{c}\text { \% de la } \\
\text { muestra }\end{array}$ & $\begin{array}{l}\text { Fuerza de las relaciones } \\
\text { (tie strength) }\end{array}$ & $\begin{array}{c}\text { N.0 de } \\
\text { empresas }\end{array}$ & $\begin{array}{c}\text { \% de la } \\
\text { muestra }\end{array}$ \\
Sinaloa (58) & 36 & 62,1 & Muy fuerte & 17 & 51,1 \\
\hline Muy fuerte & 1 & 1,7 & Fuerza media & 9 & 27,2 \\
\hline Fuerza media & 21 & 36,2 & Poco fuerte & 7 & 21,2 \\
\hline
\end{tabular}

Alta: 4,5 - 5,0; media: 2,5 - 4,49, baja: ‘2,5, de acuerdo con los índices reportados en la tabla 4.

Fuente: elaboración propia. 
Desde otro punto de vista, en Sinaloa un 37,9\% de los acuicultores mantiene relaciones con sus fuentes de conocimiento consideradas de fuerza media o baja, mientras que en Sonora este porcentaje es de 48,4 .

\section{La diversidad de los lazos de las empresas acuícolas-proveedores de conocimiento}

Como ya se mencionó antes, un aspecto relevante es la naturaleza de las relaciones que sostiene cada empresa acuícola con su red particular de fuentes de información, y especial importancia tiene el tema de la diversidad o heterogeneidad de sus contactos directos. El rango de sectores donde hemos encontrado que las empresas cultivadoras de camarón se relacionan para adquirir nuevos conocimientos técnicos es amplio: proveedores, consultores privados, empresas competidoras, universidades, tecnológicos y centros de investigación públicos, organismos gubernamentales y asociaciones civiles diversas. Una empresa tiene relaciones diversas porque sus contactos pertenecen a cada uno de estos sectores en forma acumulativa, es decir, el indicador tiene un mínimo de uno y un máximo de seis. Los resultados se muestran en las tablas 6 y 7 .

De manera comparativa, la red de Sinaloa es más diversa que la de Sonora. Sinaloa posee una mayor proporción de productores acuícolas que mantiene lazos muy diversos con las organizaciones que constituyen su red de proveedores de conocimiento técnico: el 15,5\% contra un 6,1\% en Sonora. En cambio, en Sinaloa un $84,5 \%$ de los acuicultores mantiene relaciones con sus fuentes de conocimiento consideradas de diversidad media o baja, mientras que en Sonora este porcentaje es de 94,0 .

Los estudios empíricos previos han sugerido que la heterogeneidad del conocimiento dentro de una red tiene un beneficio en el desempeño de los actores (Demirkan y Dermirkan, 2012; Huang y Liu, 2015), dado que la diversidad puede mejorar la amplitud de perspectiva, los recursos cognitivos y la capacidad de resolución de problemas.

Tabla 6. Heterogeneidad de los lazos (tie heterogeneity)) de la RFCIT en el cultivo de camarón: Sinaloa versus Sonora

\begin{tabular}{|c|c|c|c|c|c|c|c|}
\hline \multicolumn{4}{|c|}{ Sinaloa } & \multicolumn{4}{|c|}{ Sonora } \\
\hline Nodo & $\begin{array}{c}\text { índice de } \\
\text { heterogeneidad }\end{array}$ & Nodo & $\begin{array}{c}\text { índice de } \\
\text { heterogeneidad }\end{array}$ & Nodo & $\begin{array}{c}\text { índice de } \\
\text { heterogeneidad }\end{array}$ & Nodo & $\begin{array}{c}\text { índice de } \\
\text { heterogeneidad }\end{array}$ \\
\hline 01 & 5 & 30 & 3 & 01 & 3 & 30 & 3 \\
\hline 02 & 6 & 31 & 2 & 02 & 3 & 36 & 1 \\
\hline 03 & 6 & 32 & 2 & 03 & 3 & 39 & 4 \\
\hline 04 & 6 & 33 & 2 & 04 & 2 & 40 & 3 \\
\hline 05 & 4 & 34 & 2 & 05 & 2 & & \\
\hline 06 & 1 & 35 & 4 & 06 & 2 & & \\
\hline 07 & 5 & 36 & 6 & 07 & 2 & & \\
\hline 08 & 3 & 37 & 3 & 08 & 3 & & \\
\hline 09 & 2 & 38 & 2 & 09 & 2 & & \\
\hline 10 & 2 & 39 & 1 & 10 & 3 & & \\
\hline 11 & 2 & 40 & 3 & 11 & 4 & & \\
\hline 12 & 4 & 41 & 6 & 12 & 2 & & \\
\hline 13 & 3 & 42 & 3 & 13 & 6 & & \\
\hline 14 & 1 & 43 & 3 & 14 & 2 & & \\
\hline 15 & 3 & 44 & 2 & 15 & 3 & & \\
\hline 16 & 3 & 45 & 2 & 16 & 3 & & \\
\hline
\end{tabular}


Continuación Tabla 6. Heterogeneidad de los lazos (tie heterogeneity)) de la RFCIT en el cultivo de camarón: Sinaloa versus Sonora

\begin{tabular}{|c|c|c|c|c|c|c|c|}
\hline \multicolumn{4}{|c|}{ Sinaloa } & \multicolumn{4}{|c|}{ Sonora } \\
\hline Nodo & $\begin{array}{c}\text { índice de } \\
\text { heterogeneidad }\end{array}$ & Nodo & $\begin{array}{c}\text { índice de } \\
\text { heterogeneidad }\end{array}$ & Nodo & $\begin{array}{c}\text { índice de } \\
\text { heterogeneidad }\end{array}$ & Nodo & $\begin{array}{c}\text { índice de } \\
\text { heterogeneidad }\end{array}$ \\
\hline 17 & 5 & 46 & 1 & 17 & 3 & & \\
\hline 18 & 2 & 47 & 1 & 18 & 3 & & \\
\hline 19 & 2 & 48 & 2 & 19 & 2 & & \\
\hline 20 & 3 & 49 & 2 & 20 & 3 & & \\
\hline 21 & 3 & 50 & 1 & 21 & 5 & & \\
\hline 22 & 4 & 51 & 1 & 22 & 4 & & \\
\hline 23 & 4 & 52 & 3 & 23 & 4 & & \\
\hline 24 & 4 & 53 & 2 & 24 & 4 & & \\
\hline 25 & 6 & 54 & 1 & 25 & 2 & & \\
\hline 26 & 3 & 55 & 2 & 26 & 3 & & \\
\hline 27 & 4 & 56 & 2 & 27 & 3 & & \\
\hline 28 & 4 & 57 & 2 & 28 & 3 & & \\
\hline 29 & 4 & 58 & 1 & 29 & 3 & & \\
\hline
\end{tabular}

Fuente: elaboración propia.

Tabla 7. Heterogeneidad de los lazos en la RFCIT de las empresas acuícolas: Sinaloa versus Sonora

\begin{tabular}{|c|c|c|c|c|c|}
\hline $\begin{array}{l}\text { Heterogeneidad de los lazos } \\
\text { (tie heterogeneity) }\end{array}$ & $\begin{array}{l}\text { N. }{ }^{\circ} \text { de } \\
\text { empresas }\end{array}$ & $\begin{array}{l}\text { \% de la } \\
\text { muestra }\end{array}$ & $\begin{array}{l}\text { Heterogeneidad de los lazos } \\
\text { (tie heterogeneity) }\end{array}$ & $\begin{array}{c}\text { N. }{ }^{\circ} \text { de } \\
\text { empresas }\end{array}$ & $\begin{array}{l}\text { \% de la } \\
\text { muestra }\end{array}$ \\
\hline \multicolumn{3}{|c|}{ Sinaloa (58) } & \multicolumn{3}{|c|}{ Sonora (33) } \\
\hline Alta & 9 & 15,5 & Alta & 2 & 6,1 \\
\hline Media & 22 & 37,9 & Media & 21 & 63,6 \\
\hline Baja & 27 & 46,6 & Baja & 10 & 30,3 \\
\hline
\end{tabular}

Alta: 5 y 6; media: 3 y 4; baja: 1 y 2, de acuerdo con los índices reportados en la tabla 6.

Fuente: elaboración propia.

Sin embargo, otros estudios han demostrado que la diversidad puede ser un obstáculo para individuos o empresas (Schneider y Wiesehomeier, 2010, por ejemplo). Aunque la heterogeneidad puede proporcionar recursos cognitivos más amplios, también puede crear abismos o cismas que dificultan el intercambio de información. En conjunto, estos estudios recientes revelan que no se ha llegado a un entendimiento común del impacto de la diversidad de redes en el desempeño empresarial. De acuerdo con un estudio realizado para conocer la relación entre la diversidad de los contactos y la innovación de producto y proceso en las empresas acuícolas de Sonora, Gutiérrez López y León-Balderrama (2015) encontraron que la diversidad de las fuentes de conocimiento resultó una variable que influye en la innovación, ya que la diversidad de los contactos permite un mayor acceso a nuevos conocimientos y, por lo tanto, a la generación de nuevas ideas. De igual forma, de acuerdo con Fritsch y Kauffeld (2010), la diversidad puede representar un indicador de no redundancia de la información.

\section{Conclusiones}

El objetivo de este estudio ha sido analizar de manera empírica las diferencias entre Sonora y Sinaloa respecto a la configuración de las relaciones sociales que implican intercambios y flujos de conocimiento técnico en las empresas acuícolas, partiendo del estudio de la acuicultura de camarón 
desarrollada en dos de las principales regiones productoras de Sonora y Sinaloa. Particularmente se han cumplido los siguientes objetivos: a) reconstrucción para las dos regiones que cubrió el estudio de las estructuras globales de sus redes de transferencia de conocimientos, en especial las que se establecen entre las empresas acuícolas y sus fuentes de conocimiento científico y tecnológico (organismos gubernamentales, universidades, centros de investigación, proveedores, competidores, organizaciones de la sociedad civil, etc.), a través del enfoque de ARs; b) identificación de las principales características de las dos redes de transferencia de conocimiento en la industria acuícola en términos de tamaño, estructura y densidad, y análisis comparativo entre las dos entidades en estudio, y c) estimación de variables relacionales de las empresas acuícolas, por ejemplo, aquellas asociadas a la posición que ocupan dentro de la red, como el nivel de centralidad y otras que tienen que ver con las características de las relaciones que establecen con otros agentes de la red: la fuerza y la diversidad de los nexos. Con esta información se privilegió el análisis de comparación y contrastación entre las dos entidades.

Los resultados obtenidos mediante los análisis anteriores evidencian que las características y la configuración de la red de conocimiento de las industrias acuícolas de Sinaloa le permitieron a esta entidad tener una ventaja de competitividad frente a Sonora en los últimos años y resentir en menor grado los efectos de la crisis sufrida por este sector, especialmente en el periodo 2009-2015. Sinaloa tiene actores más diversos, una red menos centralizada incorpora actores novedosos como organizaciones civiles promotoras de la capacitación y la innovación. En su red las universidades y los centros de investigación juegan un papel más importante y, a diferencia de Sonora, cuenta con más diversos colaboradores o contactos internacionales.

Una de las principales limitaciones del estudio es que se basa en la percepción de gerentes de producción y propietarios de las plantas acuícolas, por lo que existen siempre sesgos en las respuestas. De igual forma, no se trata de una reconstrucción o representación exhaustiva o exacta de todos los flojos existentes, sino de una ejemplificación de su morfología y características de las relaciones con base en una muestra de tamaño limitada, pero válida para realizar ARs. Además, la investigación se enfoca en las relaciones informales y en las que implican interacción social, y de esta manera reconoce que existen flujos muy importantes de conocimientos por medios formales, que no necesariamente implican interacción social.

\section{Referencias}

Baldwin, C. y Von Hippel, E. (2011). Modeling a paradigm shift: From producer innovation to user and open collaborative innovation. Organization Science, 22(6), 1399-1417.

Berlanga-Robles, C.A., Ruiz-Luna,A.yHernández-Guzmán, R. (2011). Impact of shrimp farming on mangrove forest and other coastal wetlands: the case of Mexico. En B. Sladonja (Ed.), Aquaculture and the Environment-A Shared Destiny. IntechOpen (pp. 17-30). Rijeka, Croacia: InTech.

Booher, D. E. e Innes, J. E. (2002). Network power in collaborative planning. Journal of Planning Education and Research, 21(3), 221-236.

Borgatti, S., Everett, M. y Freeman, L. (2002). Ucinet for Windows: Software for social network analysis. Harvard, Estados Unidos: Analytic Technologies.

Borgatti, S. P., Mehra, A. Brass, D. y Labianca, G. (2009). Network analysis in the social sciences. Science, 323(5916), 892-895.

Brand, E., Gómez, H., Castaño, P., Rodríguez, J. E. y Trujillo, P. (2007). Estructura de relaciones interorganizacionales. Análisis de redes sociales Comuna 6 Medellín. La Sociología en sus Escenarios, (15).

Caloghirou, Y., Kastelli, I. y Tsakanikas, A. (2004). Internal capabilities and external knowledge sources: complements or substitutes for innovative performance? Technovation, 24(1), 29-39.

Cricelli, L. y Grimaldi, M. (2010). Knowledge-based interorganizational collaborations. Journal of Knowledge Management, 14(3), 348-358.

Choi, J., Sang-Hyun, A. y Cha, M. S. (2013). The effects of network characteristics on performance of innovation clusters. Expert Systems with Applications, 40(11), 4511-4518.

Cruz Gomes, R., Ribeiro Galina, S. V., Oliveira do Prado, F. y Silveira Porto, G. (2017). Interorganizational innovation networks of Brazilian and Spanish biotechnology companies: Dynamic comparative analysis. 
International Journal of Engineering Business Management, 9, 1-12.

D'Costa, A.P. (2002) Export growth and path-dependence: the locking-in of innovations in the software industry. Science Technology \& Society, 7(1), 51-89.

Del Río Salas, M., Martínez Durazo, A. y Jara Marini M. (2016). La acuicultura y su impacto en la zona costera del Golfo de California. Biotecnia, 18(3), 37-46.

Demirkan, I. y Demirkan, S. (2012). Network characteristics and patenting in biotechnology, 1990-2006. Journal of Management, 38(6), 1892-1927.

Enkel, E., Gassmann, O. y Chesbrough, H. (2009). Open $\mathrm{R} \& \mathrm{D}$ and open innovation: exploring the phenomenon. R\&d Management, 39(4), 311-316.

Freeman, L. C. (1979). Centrality in social networks: Conceptual clarification. Social Networks, 1(3), 215-239.

Fritsch, M. y Kauffeld-Monz, M. (2010). The impact of network structure on knowledge transfer: an application of social network analysis in the context of regional innovation networks. The Annals of Regional Science, 44(1), 21-38.

Gutiérrez, V. y León-Balderrama, J. (2015). Redes de transferencia de conocimiento y su impacto en la innovación. En Á. Bracamonte Sierra y J. I. León-Balderrama (Coords.), Redes Regionales de Conocimiento e Innovación (pp. 237-279). Hermosillo, México: El Colegio de Sonora.

Hansen, M. (1999). The search transfer problem: The role of weak ties in sharing knowledge across organization subunits. Administrative Science Quarterly, 44(1), 82-111.

Hansen, M. T., Mors, M. L. y Lovas, B. (2005). Knowledge sharing in organizations: Multiple networks, multiple phases. Academy of Management Journal, 48(5), 776-793.

Huang, C. E. y Liu, C. H. S. (2015). Employees and creativity: Social ties and access to heterogeneous knowledge. Creativity Research Journal, 27(2), 206-213.

Ibarra, H. (1993). Network centrality, power, and innovation involvement: determinants of technical and administrative roles. Academy of Management Journal, 36(3), 471-502.

Knoke, D. y Yang S. (2008). Social Network Analysis. Los Angeles, Estados Unidos: Sage.

Laursen, K. y Salter, A. (2006). Open for innovation: the role of openness in explaining innovation performance among UK manufacturing firms. Strategic Management Journal, 27(2), 131-150.

Leskovec, J. y Faloutsos, C. (agosto de 2006). Sampling from large graphs. En Proceedings of the 12th ACM SIGKDD International Conference on Knowledge
Discovery and Data Mining. Philadelphia, Estados Unidos: Asociation for Computing Machinery.

Liu, J. y Chaminade, C. (2010). Dynamics of a technological innovator network and its impact on technological performance. Innovation, 12(1), 53-74.

Lundvall, B. Å. (Ed.). (2010). National systems of innovation: Toward a theory of innovation and interactive learning (Vol. 2). Londres, Inglaterra: Anthem Press.

Maffini Gomes, C. y Kruglianskas, I. (2009) Management of external sources of technological information and innovation performance. International Journal of Innovation and Technology Management, 6(2), 207-226.

Mastrandrea, R., Fournet, J. y Barrat, A. (2015). Contact patterns in a high school: a comparison between data collected using wearable sensors, contact diaries and friendship surveys. PloS one, 10(9), e0136497.

McCarthy, B. (2008). The evolution and transformation of networks: a study of private health insurance in Ireland. Irish Journal of Management, 29(1), 87-113.

Narula, R. (2002). Innovation systems and 'inertia' in R\&D location: Norwegian firms and the role of systemic lock-in. Research Policy, 31(5), 795-816.

Nooteboom, B. (2004). Inter-firm collaboration, learning and networks. An integrated approach. London, Inglaterra: Routledge.

Oh, H., Labianca, G. y Chung, M.-H. (2006). A multilevel model of group social capital. Academy of Management Review, 31(3), 569-582

Pietrobelli, C. y R. Rabellotti (2009). Innovation systems and global value chains. En B.-A. Lundvall, K. Joseph, C. Chaminade y J. Vang (Eds.), Handbook of innovation systems and developing countries (pp. 214-238). Cheltenham, Inglaterra: Edward Elgar

Powell, W., Koput, K. y Smith-Doerr, L. (1996). Interorganizational collaboration and the locus of innovation: Networks of learning in biotechnology. Administrative Science Quarterly, 41(1), 116-145.

Reagans, R. y McEvily, B. (2003). Network Structure and Knowledge Transfer: The Effects of Cohesion and Range. Administrative Science Quarterly, 48(2), 240-67.

Rost, K. (2011). The strength of strong ties in the creation of innovation. Research Policy, 40(4), 588-604.

Scott, J. (2000) Social network analysis. A handbook, Londres, Inglaterra: Sage.

Schneider, G. y Wiesehomeier, N. (2010). Diversity, conflict and growth: Theory and evidence. Diversity, 2(9), 1097-1117.

Van der Valk, T. y Gijsbers, G. (2010). The use of social network analysis in innovation studies: Mapping actors and technologies. Innovation, 12(1), 5-17. 
Wang, H., Zhao, J., Li, Y. y Li, C. (2015). Network centrality, organizational innovation, and performance: A meta-analysis. Canadian Journal of Administrative Sciences, 32(3), 146-159.
Wasserman, S. y Faust, K. (1994). Social network analysis. Cambridge, Reino Unido: Cambridge University Press. 